\title{
DIFFUSION DEPENDENCE OF THE FITZHUGH-NAGUMO EQUATIONS
}

BY

CLYDE COLLINS

ABSTRACT. We investigate the behavior of the solutions of

$$
\begin{gathered}
u_{t}=u_{x x}-\alpha u-v+f(u), \\
v_{t}=\eta v_{x x}+\sigma u-\gamma v,
\end{gathered}
$$

as $\eta$ tends to zero from above.

1. Introduction. We consider the system of equations

$$
\left\{\begin{array}{l}
u_{t}=u_{x x}-\alpha u-v+f(u), \\
v_{t}=\eta v_{x x}+\sigma u-\gamma v,
\end{array}\right.
$$

where $(x, t) \in(0, L) \times(0, \infty)$, the parameters $(\eta, L) \in\left[0, \eta_{0}\right) \times(0, \infty]$, and $f(u)$ $=u^{2}(1+\alpha-u)$. For $\eta>0$ and $L<\infty$, we adopt the initial-boundary values

$$
\begin{gathered}
u(0, t)=g(t), \quad v(0, t)=h(t), \\
u(x, 0)=u_{0}(x), \quad v(x, 0)=v_{0}(x), \\
u_{x}(L, t)=v_{x}(L, t)=0 .
\end{gathered}
$$

When $L=\infty$ or $\eta=0$, we omit (1.4). We refer to the equations (1.1)-(1.4) or (1.1)-(1.3) as the FitzHugh-Nagumo system, abbreviated FN. When $f, g$ and $h$ are replaced by zero, the resulting equations are the homogeneous linearized FitzHughNagumo system, or HLFN.

Questions of existence, uniqueness, and boundedness of solutions of FN are considered in $[\mathbf{1}, \mathbf{5}, \mathbf{6}]$. In [1 or 2] we considered the convergence of solutions of FN for $L<\infty$ to the solution for $L=\infty$ as $L \rightarrow \infty$. The present paper addresses the convergence of solutions of FN for $\eta>0$ to the solution for $\eta=0$ as $\eta \downarrow 0$. We consider both $L=\infty$ and $L<\infty$.

As in [2] we construct resolvents for HLFN, then show resolvent consistency, which is equivalent to strong convergence of linear semigroups. We pose the versions of $\mathrm{FN}$ as operator equations involving the generators, then switch to integral equations. The convergence of linear semigroups, together with Gronwall's inequality, yields the convergence of solutions of FN.

In the case of length dependence, exponential decay rates were found. In the diffusion dependence case, decay rates will be discussed, but the results are not as strong.

Received by the editors January 24, 1983. Portions of this paper were presented at the 89th Annual Meeting of the AMS on January 7, 1983, in Denver, Colorado.

1980 Mathematics Subject Classification. Primary 35B30, 35K5j; Secondary 35C 15. 
2. Notation and technical results. First we introduce the common terms of the paper. For further explanations, see [2]. Let $s$ be the Laplace transform variable corresponding to the variable $t$. Assume $\operatorname{Re}(s)>0$. Let

$$
\begin{gathered}
M(s)=\left[\begin{array}{cc}
1 & -(s+\gamma)^{-1} \\
\sigma(s+\gamma)^{-1} & -\sigma(s+\gamma)^{-2}
\end{array}\right], \\
B=\left[\begin{array}{cc}
-\gamma & -1 \\
\sigma & -\gamma
\end{array}\right], \quad D=\left[\begin{array}{ll}
1 & 0 \\
0 & \eta
\end{array}\right], \\
U=(u, v)^{T}, \quad G(t)=(g(t), h(t))^{T}, \quad F(U)=\left(f_{1}(U), f_{2}(U)\right)^{T} .
\end{gathered}
$$

Let $a=s+\alpha, b=\sigma \eta^{-1}$ and $c=\eta^{-1}(s+\gamma)$. Define

$$
\lambda^{2}=\frac{1}{2}\left[a+c \pm \sqrt{(a-c)^{2}-4 b}\right] .
$$

Let $\lambda_{1}$ be the principal square root of $\lambda_{+}^{2} ; \lambda_{3}$ that of $\left(\lambda_{-}^{2}\right)$. Let

$$
\begin{gathered}
V_{1}=\lambda_{1}^{-1}\left(\lambda_{1}^{2}-\lambda_{3}^{2}\right)^{-2} D^{-1}\left[\begin{array}{cc}
b-\left(\lambda_{3}^{2}-a\right)^{2} & -\left(\lambda_{1}^{2}-\lambda_{3}^{2}\right) \\
{\left[b-\left(\lambda_{3}^{2}-a\right)^{2}\right]\left(\lambda_{1}^{2}-a\right)} & -\left(\lambda_{1}^{2}-a\right)\left(\lambda_{1}^{2}-\lambda_{3}^{2}\right)
\end{array}\right], \\
V_{3}=\lambda_{3}^{-1}\left(\lambda_{1}^{2}-\lambda_{3}^{2}\right)^{-2} D^{-1}\left[\begin{array}{cc}
b-\left(\lambda_{1}^{2}-a\right)^{2} & \lambda_{1}^{2}-\lambda_{3}^{2} \\
{\left[b-\left(\lambda_{1}^{2}-a\right)^{2}\right]\left(\lambda_{3}^{2}-a\right)} & \left(\lambda_{3}^{2}-a\right)\left(\lambda_{1}^{2}-\lambda_{3}^{2}\right)
\end{array}\right] .
\end{gathered}
$$

For $L<\infty$, define for either $i=1$ or $i=3$,

$$
k_{i}^{\eta}(x, y, s)= \begin{cases}\sinh \left(\lambda_{i} x\right) \cosh \left(\lambda_{i}(L-y)\right)\left[\cosh \left(\lambda_{i} L\right)\right]^{-1}, & x<y, \\ \sinh \left(\lambda_{i} y\right) \cosh \left(\lambda_{i}(L-x)\right)\left[\cosh \left(\lambda_{i} L\right)\right]^{-1}, & y<x .\end{cases}
$$

Let $f(s)=a+\sigma \eta c=s+\alpha+\sigma(s+\gamma)^{-1}$. Define

$$
k(x, y, s)= \begin{cases}\sinh (x \sqrt{f(s)}) \cosh ((L-y) \sqrt{f(s)})[\cosh (L \sqrt{f(s)})]^{-1}, \quad x<y, \\ \sinh (y \sqrt{f(s)}) \cosh ((L-x) \sqrt{f(s)})[\cosh (L \sqrt{f(s)})]^{-1}, \quad y<x .\end{cases}
$$

For $L=\infty$, define for $\eta>0$ and either $i=1$ or $i=3$,

$$
j_{i}^{\eta}(x, y, s)= \begin{cases}\sinh \left(\lambda_{i} x\right) e^{-\lambda_{i} y}, & x<y, \\ \sinh \left(\lambda_{i} y\right) e^{-\lambda_{i} x}, & y<x .\end{cases}
$$

For $\eta=0, L=\infty$, define

$$
j(x, y, s)= \begin{cases}\sinh (x \sqrt{f(s)}) e^{-y \sqrt{f(s)}}, & x<y, \\ \sinh (y \sqrt{f(s)}) e^{-x \sqrt{f(s)}}, & y<x .\end{cases}
$$

Further, let

$$
\begin{aligned}
J^{\eta}(x, y, s) & =j_{1}^{\eta}(x, y, s) V_{1}+j_{3}^{\eta}(x, y, s) V_{3}, \\
J(x, y, s) & =f(s)^{-1 / 2} J(x, y, s) M(s),
\end{aligned}
$$




$$
\begin{aligned}
G^{\eta}(x, y, s) & =k_{1}^{\eta}(x, y, s) V_{1}+k_{3}^{\eta}(x, y, s) V_{3}, \\
G_{1}(x, y, s) & =f(s)^{-1 / 2} k(x, y, s) M(s) .
\end{aligned}
$$

Applying the Laplace transform to HLFN and following by rearrangement, we obtain, for $\eta>0$,

$$
\left\{\begin{array}{l}
\bar{u}_{x x}=a \bar{u}+\bar{v}-u_{0}, \\
\bar{v}_{x x}=-b \bar{u}+c \bar{v}-v_{0} .
\end{array}\right.
$$

For $\eta=0$, transformation and rearrangement yields

$$
\left\{\begin{array}{l}
\bar{u}_{x x}=a \bar{u}+\bar{v}-u_{0}, \\
0=-\sigma \bar{u}+(s+\gamma) \bar{v}-v_{0} .
\end{array}\right.
$$

Solving (2.1) yields the functions $\lambda_{1}(s)$ and $\lambda_{3}(s)$. When $L=\infty$, the solution of (2.1) is given by

$$
\bar{U}^{\eta}(x, s)=\int_{0}^{\infty} J^{\eta}(x, y, s) U_{0}(y) d y .
$$

For $L<\infty$, the solution of (2.1) is given by

$$
\bar{U}_{L}^{\eta}(x, s)=\int_{0}^{L} G^{\eta}(x, y, s) U_{0}(y) d y .
$$

The solution of (2.2) for $L=\infty$ is given by

$$
\bar{U}(x, s)=\int_{0}^{\infty} J(x, y, s) U_{0}(y) d y+\left(0, v_{0}(x)(s+\gamma)^{-1}\right)^{T},
$$

whereas for $L<\infty$, the solution of (2.2) is given by

$$
\bar{U}_{L}(x, s)=\int_{0}^{L} G(x, y, s) \bar{U}_{0}(y) d y+\left(0, v_{0}(x)(s+\gamma)^{-1}\right)^{T} .
$$

In [2], we showed that $\bar{U}_{L}^{\eta} \rightarrow \bar{U}^{\eta}$ in $C^{0}\left(0, \xi L ; R^{2}\right)$ for $\xi \in(0,1)$. That is, the resolvents converged strongly. In the present paper, we show that $\bar{U}^{\eta} \rightarrow \bar{U}$ in $C^{0}\left(0, \infty ; R^{2}\right)$ and $\bar{U}_{L}^{\eta} \rightarrow \bar{U}_{L}$ in $C^{0}\left(0, L ; R^{2}\right)$. To do this, we need some technical results. We list some from [1]:

(i) $\operatorname{Re}\left(\lambda_{1}\right) \geqslant[\gamma / 2 \eta]^{1 / 2}$ for any fixed $\operatorname{Re}(s)>0$, for all $\operatorname{Im}(s) \in R$.

(ii) $\left(\lambda_{1}\right) \rightarrow \infty$ as $\eta \downarrow 0$.

(iii) $b-\left(\lambda_{1}^{2}-a\right)^{2}=-\left(\lambda_{1}^{2}-a\right)\left(\lambda_{1}^{2}-\lambda_{3}^{2}\right)$.

(iv) $\left[b-\left(\lambda_{1}^{2}-a\right)^{2}\right]\left(\lambda_{3}^{2}-a\right)=-b\left(\lambda_{1}^{2}-\lambda_{3}^{2}\right)$.

(v) $b-\left(\lambda_{3}^{2}-a\right)^{2}=\left(\lambda_{3}^{2}-a\right)\left(\lambda_{3}^{2}\right)$.

Further, a little algebra shows that

(vi) $\lambda_{3} \rightarrow \sqrt{f(s)}$ as $\eta \downarrow 0$.

(vii) $\eta\left(\lambda_{1}^{2}-\lambda_{3}^{2}\right) \rightarrow s+\gamma$ as $\eta \downarrow 0$.

3. Resolvent consistency and rates. Using (i)-(vii) of $\S 2$, a few calculations show that

$$
V_{3} \rightarrow f(s)^{-1 / 2} M(s), \quad j_{3}^{\eta}(x, y, s) \rightarrow j(x, y, s), \quad k_{3}^{\eta}(x, y, s) \rightarrow k(x, y, s) .
$$


Further, (ii) of $\S 2$ shows that the $(1,1),(1,2)$ and $(2,1)$ entries of $V_{1}$ go to zero as $\eta \downarrow 0$. Since $\left\|j_{1}^{\eta}(x, \cdot, s)\right\|$ and $\left\|k_{1}^{\eta}(x, \cdot, s)\right\|$ are bounded, the action of these components of the kernels $J^{\eta}$ and $K^{\eta}$ go to zero as $\eta \downarrow 0$. The $(2,2)$ entry of $k_{1}^{\eta} V_{1}$ is

$$
{ }^{-1} \eta^{-1} \lambda_{1}^{-1}\left(\lambda_{1}^{2}-\lambda_{3}^{2}\right)^{-1}\left(\lambda_{1}^{2}-a\right) k_{1}^{\eta}(x, y, s) \text {. }
$$

For $\eta>0, \int_{0}^{L} \lambda_{1} k_{1}^{\eta}(x, y, s) d y=1-\left[\cosh \left(\lambda_{1} L\right)\right]^{-1} \cosh \lambda_{1}(L-x)$; the integral converges to 1 as $\eta \downarrow 0$ except at $x=0$. Let $Q$ be the difference between the $(2,2)$ entry of $k_{1}^{\eta} V_{1}$ and $v_{0}(x)[s+\gamma]^{-1}$. Then

$$
\begin{aligned}
Q= & \int_{0}^{l}\left[\lambda_{1}^{-1}\left(\lambda_{1}^{2}-a\right)\right]\left[\eta^{-1}\left(\lambda_{1}^{2}-\lambda_{3}^{2}\right)\right] \cdot \lambda_{1} \cdot k_{1}^{\eta}(x, y, s) v_{0}(y) d y \\
& -\int_{0}^{l} \lambda_{1} k_{1}^{\eta}(x, y, s) v_{0}(x)(s+\gamma)^{-1} d y \\
& -\left[v_{0}(x) \cosh \lambda_{1}(L-x)\right]\left[(s+\gamma) \cosh \lambda_{1} L\right]^{-1} \\
= & \int_{0}^{L} \lambda_{1} k_{1}^{\eta}(x, y, s)\left[v_{0}(y)-v_{0}(x)\right](s+\gamma)^{-1} d y+\delta(\eta, x)
\end{aligned}
$$

where

$$
\begin{aligned}
\delta(\eta, x)= & {\left[\lambda_{1}^{-2}\left(\lambda_{1}^{2}-a\right)\left(\eta^{-1}\left(\lambda_{1}^{2}-\lambda_{3}^{2}\right)^{-1}\right)-(s+\gamma)^{-1}\right] \int_{0}^{L} \lambda_{1} k_{1}^{\eta}(x, y, s) v_{0}(y) d y } \\
& -v_{0}(x) \cosh \lambda_{1}(L-x)(s+\gamma)^{-1}\left(\cosh \lambda_{1} L\right)^{-1} .
\end{aligned}
$$

Since

$$
\begin{aligned}
|\delta(\eta, x)| \leqslant & \left|\lambda_{1}^{-2}\left(\lambda_{1}^{2}-a\right) \eta^{-1}\left(\lambda_{1}^{2}-\lambda_{3}^{2}\right)^{-1}-(s+\gamma)^{-1}\right| \cdot\left\|v_{0}\right\|_{\infty} \\
& +\left\|v_{0}\right\|_{\infty}|s+\gamma|^{-1}\left|\cosh \lambda_{1}(L-x)\left(\cosh \lambda_{1} L\right)^{-1}\right|
\end{aligned}
$$

we see that $\delta(\eta, x) \rightarrow 0$ uniformly in $x$ as $\eta \downarrow 0$. (Recall that $v_{0}(x)=0$ at $x=0$.) Further,

$$
\begin{aligned}
& \lambda_{1}^{-2}\left(\lambda_{1}^{2}-a\right) \eta^{-1}\left(\lambda_{1}^{2}-\lambda_{3}^{2}\right)^{-1}-(s+\gamma)^{-1} \\
& \quad=\left(\eta^{-1}\left(\lambda_{1}^{2}-\lambda_{3}^{2}\right)^{-1}-(s+\gamma)^{-1}\right)-a \cdot \lambda_{1}^{-2} \eta^{-1}\left(\lambda_{1}^{2}-\lambda_{3}^{2}\right)^{-1} .
\end{aligned}
$$

Since $\eta\left(\lambda_{1}^{2}-\lambda_{3}^{2}\right) \rightarrow s+\gamma$ and (i) of $\S 2$ holds, the second term above goes to zero as $C \eta$ for $\eta \rightarrow 0$. Algebra shows that the first term equals

$\eta\left(2(s+\alpha)(s+\gamma)+4 \sigma-\eta(s+\alpha)^{2}\right)\left((s+\gamma) \eta\left(\lambda_{1}^{2}-\lambda_{3}^{2}\right)\left(s+\gamma+\eta\left(\lambda_{1}^{2}-\lambda_{3}^{2}\right)\right)\right)^{-1}$.

The denominator converges to $2(s+\gamma)^{3}$ while the numerator is bounded away from 0 and $\infty$ as $\eta \downarrow 0$. Hence the first term is proportional to $\eta$ as $\eta$ vanishes.

The quotient $\cosh \lambda_{1}(L-x)\left[\cosh \left(\lambda_{1} L\right)\right]^{-1}$ has the value 1 at $x=0$ and the value $\left(\cosh \lambda_{1} L\right)^{-1}$ at $x=L$. Outside any neighborhood of zero, the quotient decays exponentially as $\eta \downarrow 0$. This, together with the fixed value of 1 at $x=0$, shows us we have a "spine" at $x=0$. However, for $v_{0} \equiv 0$ in a neighborhood of $x=0$ or for $\left|v_{0}(x)\right| \leqslant k e^{-h / x}$ for $x \downarrow 0$, the second term of $\delta(\eta, x)$ goes to zero exponentially as $\eta \downarrow 0$. 
However, the integral

$$
m(x)=\int_{0}^{L} \lambda_{1} k_{1}^{\eta}(x, y, s)\left[v_{0}(y)-v_{0}(x)\right] d y
$$

is more delicate. Let $\varepsilon>0$ be given. Suppose $|x-y| \geqslant \varepsilon$. Since $0 \leqslant\left|k_{1}^{\eta}(x, y, s)\right| \leqslant$ $2 e^{-\varepsilon \gamma / \sqrt{\eta}}$ for these $x$ and $y$, we have that

$$
\begin{aligned}
|m(x)| \leqslant\left|\int_{x-\varepsilon}^{x+\varepsilon} \lambda_{1} k_{1}^{\eta}(x, y, s) d y\right| \cdot \sup _{|x-y|<\varepsilon}\left|v_{0}(y)-v_{0}(x)\right|+4\left\|v_{0}\right\|_{\infty} e^{-\varepsilon \gamma / 2 \sqrt{\eta}} \\
\quad \leqslant 2 \sup _{|x-y|<\varepsilon}\left|v_{0}(y)-v_{0}(x)\right|+4\left\|v_{0}\right\|_{\infty} e^{-\varepsilon \gamma / 2 \sqrt{\eta}} .
\end{aligned}
$$

For $v_{0} \in C^{0}$, we get no decay rate. However, if $v_{0} \in \operatorname{Lip}(\zeta), \zeta \in(0,1]$, then $|m(x)| \leqslant 2 C \varepsilon^{\zeta}+4\left\|v_{0}\right\|_{\infty} e^{-\varepsilon \gamma / 2 \sqrt{\eta}}$. For $\varepsilon=\eta^{r}$, we have

$$
|m(x)| \leqslant 2 C \eta^{r \zeta}+4\left\|v_{0}\right\|_{\infty} \exp \left(-\gamma \eta^{r-1 / 2}\right) / 2 .
$$

Lemma 3.1. Let $v_{0} \in \operatorname{Lip}(\zeta)$ for $0 \leqslant \zeta \leqslant 1$. Then $Q$ goes to zero proportional to $\eta^{\omega}$, for $\omega=r \zeta, 0<r<1 / 2$, provided that $v_{0}(x) \rightarrow 0$ as $e^{-k / x}$ for $x \downarrow 0$.

Proof. Note that $\zeta=0$ refers to uniform convergence without decay rate.

THEOREM 3.2. The decay rates for the convergence of

$$
\int_{0}^{L} G^{\eta}(x, y, s)\left(u_{0}(y), v_{0}(y)\right)^{T} d y \rightarrow \int_{0}^{L} G(x, y, s)\left(u_{0}(y), v_{0}(y)\right)^{T} d y
$$

and

$$
\int_{0}^{\infty} J^{\eta}(x, y, s)\left(u_{0}(y), v_{0}(y)\right)^{T} d y \rightarrow \int_{0}^{\infty} J(x, y, s)\left(u_{0}(y), v_{0}(y)\right)^{T} d y
$$

are as follows:

(i) uniform convergence, if $v_{0} \in C^{0}-\operatorname{Lip}(\zeta)$ for $\zeta>0$ or $v_{0}$ does not go to zero exponentially as $x \downarrow 0$.

(ii) $C_{1} \eta^{r \zeta}$ if $v_{0} \in \operatorname{Lip}(\zeta), 0<r<1 / 2$, and $v_{0}(x) \rightarrow 0$ as $e^{-k / x}$ as $x \downarrow 0$.

(iii) $C_{2} \eta$ if $v_{0} \equiv 0$.

Proof. A routine analysis of $k_{3}^{\eta}(x, y, s) V_{3} \rightarrow f^{-1 / 2}(s) k(x, y, s) M(s)$ shows that the decay rate is $C_{3} \eta$. With $v_{0} \equiv 0$, we have (iii). Lemma 3.1, coupled with (iii), yields (i) and (ii). For $L=\infty$, the proofs are isomorphic.

As in [1, 2], we consider HLFN as an operator equation

$$
U_{t}^{\eta}=\left(A^{\eta}+B\right) U^{\eta}, \quad U^{\eta}(0)=U_{0},
$$

with solution $U^{\eta}(t)=T^{\eta}(t) U_{0}$. Taking Laplace transforms, we get

$$
\bar{U}^{\eta}(s)=L\left[T^{\eta}(t)\right](s) U_{0}=R\left(s, A^{\eta}+B\right) U_{0} .
$$

The resolvent $R\left(s, A^{\eta}+B\right)$ we have represented by kernels for $\eta \geqslant 0, L \leqslant \infty$. Theorem 3.2 shows that $R\left(s, A^{\eta}+B\right) \rightarrow R(s, A+B)$ in the strong operator topology as $\eta \downarrow 0$ for $L \leqslant \infty$. That is, resolvent consistency is shown with the decay rates given above. 
4. Linear semigroup convergence. The resolvent consistency shown in $\S 3$ implies semigroup consistency [4, Theorem 2.16]. Hence $T^{\eta}(t) \stackrel{s}{\rightarrow} T(t)$ in $C^{0}\left(0, L ; R^{2}\right)$ uniformly in $t \in[0, T]$. Since $\operatorname{Re}\left(\sigma\left(A^{L, \eta}+B\right)\right)<-\delta=-\min (\alpha, \gamma) / 2<0$ independent of $L \leqslant \infty$ and independent of $\eta \geqslant 0$, we have $\left\|T^{\eta}(t)\right\| \leqslant C \cdot e^{-\delta t}$. Hence $T^{\eta}(t)=\int_{c-i \infty}^{c+i \infty} e_{t}^{s t} T\left(s, A^{\eta}+B\right) d s$ converges for $\operatorname{Re}(c) \geqslant-\delta$. Further,

$$
\left\|\left(T^{\eta}(t)-T(t)\right) U_{0}\right\| \leqslant\left|\int_{c-i \infty}^{c+i \infty} e^{s t} d s\right| \cdot\left\|\left(R\left(s, A^{\eta}+B\right)-R(s, A+B)\right) U_{0}\right\| .
$$

Our earlier work then yields decay estimates for $\left\|\left(T^{n}(t)-T(t)\right) U_{0}\right\|$ for $t>0$.

5. Convergence of solutions of the FitzHugh-Nagumo equations. The Rauch-Smoller theory in $[1,2,5,6]$ gives a priori estimates for $\mathrm{FN}$. We make the change variable $W=(w, z)^{T}=U-G=(u, v)^{T}-(g, h)^{T}$. Then FN becomes

$$
D_{t} W=\left(D D_{x x}+B\right) W+K(t, w)
$$

subject to

$$
\begin{array}{ll}
W(0, t)=(0,0)^{T}, & 0<t<\infty, \\
D_{x} W(L, t)=(0,0), & L<\infty \text { only, } 0<t<\infty, \\
W(x, 0)=U_{0}(x), & 0 \leqslant x \leqslant L,
\end{array}
$$

where $K(t, w)=B G-D_{t} G+\left((w+g)^{2}(1+\alpha-w-g), 0\right)^{T}$. The theory of Henry $[2,3]$, yields the equivalence of $(5.1)$ to the integral equation

$$
W^{\eta}(t)=T^{\eta}(t) W_{0}^{\eta}+\int_{0}^{t} T^{\eta}(t-s) K\left(s, W^{\eta}(s)\right) d s
$$

or

$$
W(t)=T(t) W_{0}+\int_{0}^{t} T(t-s) K(s, W(s)) d s .
$$

Let $Z(t)=W^{\eta}(t)-W(t)$; let $r(\eta)$ be a quantity that vanishes (with decay rates as in Theorem 3.2).

THEOREM 5.1. There exist constants $c_{1}, c_{2}, c_{3}$ and $\delta>0$ such that

$$
\|Z(t)\| \leqslant\left[c_{1}\left\|Z_{0}\right\|+r(\eta) c_{2} \exp (\delta t) \exp \left(c_{3} T_{1}\right)\right]
$$

for $t \in\left[0, T_{1}\right]$; the norm is that of $C^{0}\left(0, L ; R^{2}\right)$, for $L \leqslant \infty$.

Proof. Subtraction of (5.3) from (5.2) yields

$$
\begin{aligned}
Z(t)= & \left(T^{\eta}-T\right)(t) W_{0}^{\eta}+\int_{0}^{t}\left(T^{\eta}-T\right)(t-s) K\left(s, W^{\eta}(s)\right) d s \\
& +T(t) Z_{0}+\int_{0}^{t} T(t-s)\left[K\left(s, W^{\eta}(s)\right)-K(s, W(s))\right] d s .
\end{aligned}
$$

We estimate the last equation

$$
\begin{aligned}
\|Z(t)\| \leqslant & r(\eta)+t \cdot r(\eta)+C \cdot \exp (-\delta \tau)\left\|Z_{0}\right\| \\
& +\int_{0}^{t} M C \cdot \exp (-\delta[t-s])\|Z(s)\| d s
\end{aligned}
$$


as $\eta \downarrow 0$, where $M$ is the Lipschitz constant for $K(s, W)$. The above inequality becomes

$$
\begin{aligned}
\|Z(t)\| \exp (\delta t) \leqslant & {[1+t] r(\eta) \exp (\delta t)+C\left\|Z_{0}\right\| } \\
& +\int_{0}^{t} C M \exp (\delta s)\|Z(s)\| d s .
\end{aligned}
$$

Applying Gronwall's inequality, we obtain

$$
\begin{aligned}
\|Z(t)\| & \leqslant\left[C\left\|Z_{0}\right\|+(1+t) r(\eta) \exp (\delta t)\right] \exp [(M C-\delta) t] \\
& \leqslant\left[C \cdot\left\|Z_{0}\right\|+\left(1+T_{1}\right) r(\eta) \exp (\delta t)\right] \exp \left[(M C-\delta) T_{1}\right]
\end{aligned}
$$

for $t \in\left[0, T_{1}\right)$.

COROllaRy 5.2. If $\left\|Z_{0}\right\|=0$, then $\|Z(t)\| \rightarrow 0$ as $\eta \downarrow 0$, uniformly in $t \in\left[0, T_{1}\right]$.

Proof. $\left\|Z_{0}\right\|=0$ implies $r(\eta)$ is a multiplier of the entire right-hand side of (5.4).

Remark. $W^{\eta} \rightarrow W$ implies $U^{\eta} \rightarrow U$ in $C\left(0, L ; R^{2}\right), L \leqslant \infty$, uniformly in $\left[0, T_{1}\right]$. Hence we have convergence of our original functions.

REMARK. The work of this paper connects the solutions of FN with $\eta=0$ with those for $\eta>0$. For "nice" initial conditions, decay rates are given. As remarked in $[1,2]$, the initial condition $U_{0}(x)=(0,0)^{T}$ is a very fitting one, and certainly satisfied our definition of "nice" discussed above. Hence the best decay rate applies.

\section{REFERENCES}

1. C. Collins, Length dependence of solutions of generalized FitzHugh-Nagumo equations, Ph.D. Thesis, Indiana Univ., Bloomington, 1981.

2. L Length dependence of solutions of FitzHugh-Nagumo equations, Trans. Amer. Math. Soc. 280(1983), 809-832.

3. D. Henry, Geometric theory of semilinear parabolic equations, Springer-Verlag, New York, 1981.

4. T. Kato, Perturbation theory for linear operators, Springer-Velag, New York, 1980.

5. J. Rauch and J. Smoller, Qualitative theory of the FitzHugh-Nagumo equations, Adv. in Math. 27 (1978), 12-44.

6. M. Schonbek, Technical reports 1739 and 1740, MRC, Madison, Wisconsin, 1977.

Department of Mathematics, Louisiana State University, Baton Rouge, Louisiana 70803 